\title{
Posterior Fixation with Unilateral Same Segment Pedicle Fixation and Contralateral Hook in Surgical Treatment of Thoracolumbar Burst Fractures
}

\author{
Farzad Omidi-Kashani, ${ }^{1,}$ Ebrahim Ghayem Hasankhani, ${ }^{1}$ Mahmoud Tavousi, ${ }^{1}$ Amir Ashjazadeh, ${ }^{1}$ and \\ Mohammad Hosein Ebrahimzadeh ${ }^{1}$ \\ ${ }^{1}$ Department of Orthopedic, Orthopedic Research Center, Imam Reza Hospital, Mashhad University of Medical Sciences, Mashhad, Iran \\ "Corresponding author: Farzad Omidi Kashani, Department of Orthopedic, Orthopedic Research Center, Imam Reza Hospital, Mashhad University of Medical Sciences, \\ Mashhad, Iran. E-mail: omidif@mums.ac.ir
}

Received 2015 April 29; Accepted 2015 September 15.

\begin{abstract}
Background: In surgical treatment of thoracolumbar burst fractures, most authors try to lower the number of vertebrae involved during the surgery.

Objectives: The aim of this study was to evaluate the outcome of a medium-segment posterior spinal fixation in these patients. Patients and Methods: We retrospectively reviewed 27 patients (18 male, 9 female) with mean age of $39.4 \pm 15.0$ years old in a before-and-after study. The mean follow-up period was $38.4 \pm 15.6$ months. We involved 2 intact above vertebrae and one intact below vertebra, inserting a pedicular screw at the fractured level and supplemented the construct with contralateral infralaminar hook. Clinical and radiologic characteristics were assessed with American spinal injury association (ASIA) scale, oswestry disability index (ODI), visual analogue scale (VAS), and plain radiography. Data analysis was carried out by SPSS version 11.5 software.

Results: Mean post traumatic kyphosis was $+15.7^{\circ} \pm 3.3^{\circ}$ that was changed to $-8.5^{\circ} \pm 4.3^{\circ}$ and $+1^{\circ} \pm 4.4^{\circ}$ at immediate and last visit after surgery, respectively. Mean loss of correction (LOC) was $9.5^{\circ} \pm 1.9^{\circ}(\mathrm{P}<0.001)$. At the most recent follow-up visit, mean ODI and VAS were $15.0 \pm 14.4$ and $2.4 \pm 2.5$, respectively and 24 cases (88.9\%) declared excellent or good clinical results. At the last follow-up visit, LOC had no significant correlation neither with VAS nor ODI.

Conclusions: In surgical treatment of thoracolumbar burst fractures, a medium-segment posterior spinal fixation, although cannot maintain the radiologic reduction of the fractured vertebrae efficiently, is not only associated with acceptable clinical outcome but also spare one lower intact lumbar segment and therefore recommended.
\end{abstract}

Keywords: Spinal Fractures Instrumentation, Pedicle Screws, Spinal Fusion

\section{Background}

Thoracolumbar area is the most common location for spinal fracture and its optimal management comprises one of the most controversial areas in spine surgery [1-3]. Although some authors believe that in more stable thoracolumbar burst fractures associated with normal neurologic examination, nonoperative treatment provided the same long-term advantages as surgery, in the patients with more severe injuries and neurologic deficit, early fusion and instrumentation can promote earlier mobilization and neurologic rehabilitation [4-7]. In spinal surgery of lumbar area, literature reveals that spondylodesis of the freely moving lumbar vertebrae is strongly correlated with functional disability in activities of daily living [8]. Therefore, in surgical treatment of thoracolumbar burst fractures, most authors try to lower the number of lumbar vertebrae involved during the surgery. In order to do this, several proposals have been tried including anterior fusion and instrumentation, or short posterior instrumentation with or without anterior reconstruction and fusion [9-12]. Each of these surgical options has its own cons and pores. In this study, we tried to spare one inferior lumbar vertebra by inserting one pedicular screw at the level of injury and supplementing it by a contralateral infralaminar hook at the inferior intact lumbar vertebra.

\section{Objectives}

The aim of this study is to evaluate the clinical and radiologic outcome of those patients who have been treated by this kind of posterior spinal fusion and instrumentation for thoracolumbar burst fractures.

\section{Patients and Methods}

After local institutional review board approval (Code number 920630) we arranged a before-and-after study and 
retrospectively reviewed our patients with thoracolumbar burst fracture who had been surgically treated by unilateral pedicle fixation at the level of fracture associated with contralateral supplementary laminar hook. From September 2008 to October 2013, 34 cases with unstable thoracolumbar burst fracture were operated in our orthopedic department. Our inclusion criteria were burst fractures occurring from T10 to L2 with a post-traumatic kyphosis > $15^{\circ}$, canal compromise $>50 \%$, vertebral height loss $>50 \%$, or three column vertebral failure [13-16]. Those cases with significant neurologic deficit associated with severely displaced anterior bony retropulsion were treated from anterior approach and these patients were excluded from our study. We also excluded fracture age more than three weeks, follow-up period less than two years, and significant underlying disease like uncontrolled diabetes mellitus, osteoporosis, hemorrhagic diathesis, and pathologic fractures. Ultimately, 27 patients were found eligible and entered the study. The fractures' morphology and the patients' neurologic status were classified according to Dennis and American spinal injury association (ASIA) scale, respectively. $[17,18]$. Post-traumatic kyphosis was assessed by drawing the lines parallel with superior and inferior endplates of the intact adjacent upper and lower vertebrae, respectively. At the last follow-up visit, disability and pain were separately evaluated. oswestry disability index (ODI) questionnaire version 2.1 was used to estimate the disability, while back pain was measured on a numerical scaling line (visual analogue scale; VAS) from zero to ten. These questionnaires were already translated and validated for Iranian patients [19-21]. All the patients signed the informed consent and were aware from pros and cons of this surgical procedure by the surgical team. Throughout this period, all surgical procedures were carried out by the senior author (Farzad Omidi-Kashani) with the same instrument system (Xia Stryker Corporation, US) and similar fusion technique (a mixture of local bone graft and freeze dried cortical cancellous matchstick allograft taken from tissue regeneration corporation; TRC, Kish, Iran).

\subsection{Surgical Technique}

In all of the patients, primary closed reduction before skin incision was carried out by gentle trunk hyperextension while the patient placed in prone position. Gentle intraoperative distraction and regional lordosis restoration were also performed. In this modified technique, we instrumented two intact upper vertebrae but spared one intact lower lumbar vertebra with inserting one pedicular screw into the fractured vertebra and adding one contralateral infralaminar hook at the intact inferior adjacent vertebra (Figure 1). We used neither laminectomy nor direct fracture reduction in this study. Transverse connec- tors were uniformly added at the level of the fractured vertebra in all the cases. Postoperatively, the patients were ambulated as soon as possible (usually one or two days after surgery) while worn a soft thoracolumbar orthosis for added support. The patients usually discharged from the hospital on the day 2 or 3 , after they were able to walk and void independently. They were followed-up postoperatively at 4 weeks, 3, 6, 12, and 24 months with careful clinical and radiological assessment. All significant intraand postoperative complications were also recorded. Computed tomography was not used routinely except in the patients with suspicious pseudoarthrosis or screw malpositioning. At the most recent follow-up visit, patients' satisfaction with surgery was assessed according to criteria derived from the North American spine society low back outcome instrument and rated as excellent, good, fair, or poor [22].

\subsection{Statistical Analysis:}

Data analysis was carried out by SPSS (statistical package for social sciences) Chicago, IL, US, version 11.5. Normal distribution of the data was confirmed by KolmogorovSmirnov test. Independent $\mathrm{T}$ test or ANOVA (analysis of variance) and paired $\mathrm{T}$ test were used to compare quantitative and dependent variables, respectively. Correlation between the variables was analyzed by Pearson test. Level of significance was set as $\mathrm{P}<0.05$.

\section{Results}

Seven patients were excluded from the study due to lack of necessary criteria. Demographic data of the both groups of patients were shown in Table 1. No significant intra- or postoperative complication occurred in our patients. Screw breakage, loosening or pull-out necessitated revision surgery did not happen. We had no patient with suspicious pseudoarthrosis necessitated computed tomography scanning. Superficial wound infection occurred in two patients that were treated with local wound care and oral antibiotics. Radiologic and clinical characteristics of the patients at the time of presentation, immediately after surgery, and at the last follow-up visits were also depicted in detail in Table 2. Mean follow-up period was $38.4 \pm 15.6$ (ranged; 25 - 72) months. Loss of correction (LOC) throughout this follow-up period was $9.5 \pm 1.9$ (ranged 6 - 14) degrees. This amount of LOC was significant statistically $(\mathrm{P}<0.001)$ without any different between male and female patients $(\mathrm{P}=0.477)$. At the last follow-up visit, LOC had no significant correlation neither with VAS nor ODI $(\mathrm{P}=0.254, \mathrm{r}=0.228$ and $\mathrm{P}=0.139, \mathrm{r}=0.299$, respectively). None of our patients had poor surgical outcome 

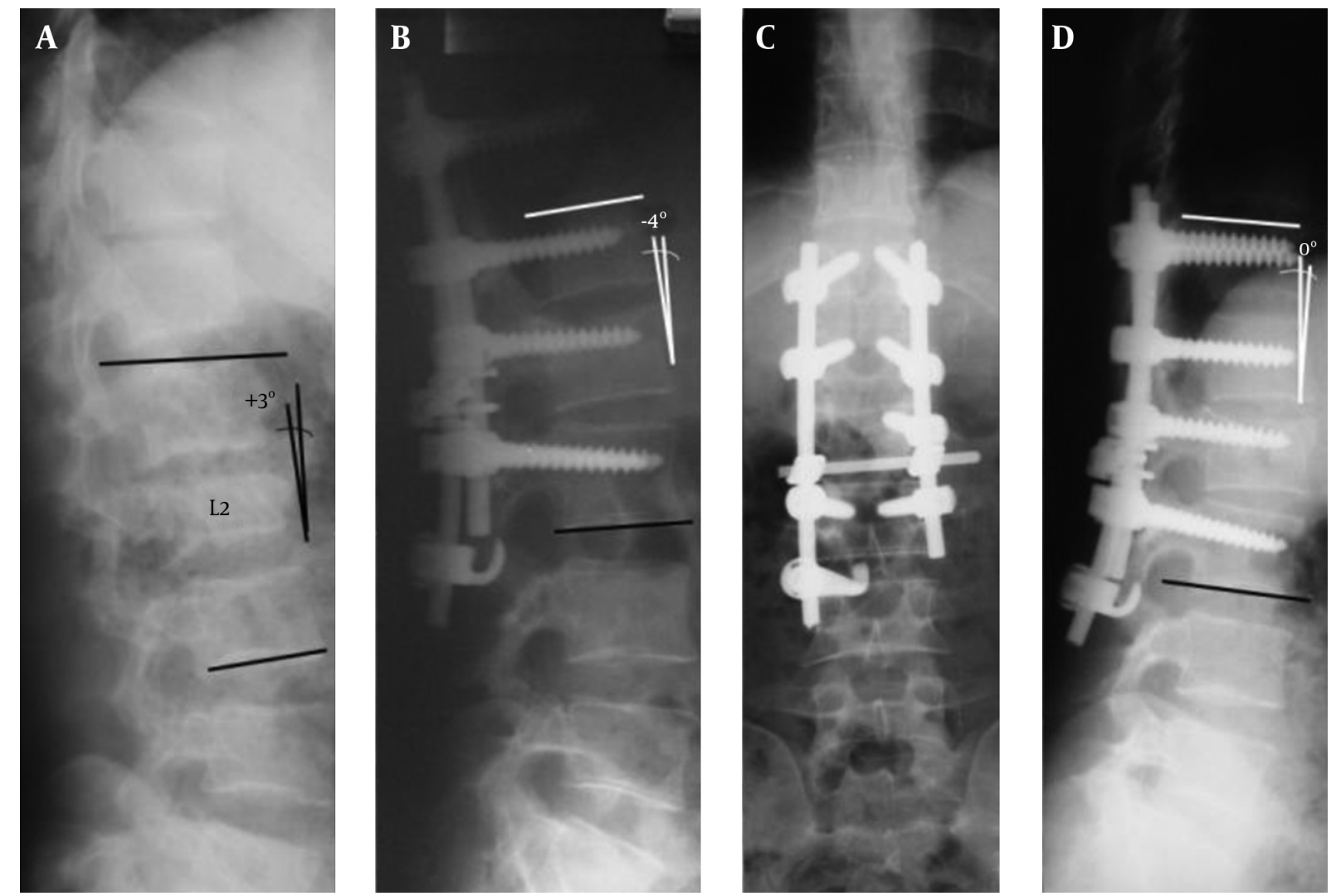

Figure 1. A, a 29 years old woman with L2 burst fracture, on Primary radiography; B, significant vertebral body collapse was observed due to three columns failure, significant height restoration was achieved on immediate postoperative view; $C$ and D, remained relatively constant throughout the follow-up period of 41 months

and 24 (88.9\%) declared either excellent or good clinical results.

\section{Discussion}

Our study showed that posterior fusion and instrumentation with unilateral fracture site screw insertion and contralateral infralaminar hook, although could not maintain the radiologic reduction efficiently, it was associated with good or excellent clinical outcome. Literature declares that the term of "short segment posterior fixation" is usually applied for pedicle screw fixation from only one level above to one level below the fractured segment. In the study we carried out, a medium-segment posterior fixation was really performed. In surgical treatment of thoracolumbar burst fractures, loss of correction is the main concern of the surgeons dealing with short segment posterior fixation. This loss of correction may be attributed to implant failure (bending or breakage of the screw or rod), underlying pathology (like osteoporosis), inappropriate instrumentation (incorrect screw insertion, small screw
Table 1. Demographic Data of Our Treated Patients at the Time of Presentation

\begin{tabular}{lc}
\hline Characteristics & Values \\
\hline Number & 27 \\
\hline Age, mean \pm SD (range) & $39.4 \pm 15.0(19-68)$ \\
\hline Gender, male/female & $18 / 9$ \\
\hline Height, cm, mean \pm SD (range) & $169.4 \pm 8.1(150-184)$ \\
\hline Weight, kg, mean \pm SD (range) & $73.8 \pm 10.3(51-110)$ \\
\hline Fracture level & 1 \\
\hline$-T 10$ & 2 \\
\hline T11 & 6 \\
\hline -T12 & 10 \\
\hline -L1 & 4 \\
\hline -L2 & 2 \\
\hline -T12 \& L1 & 1 \\
\hline -L1 \& L2 & 1 \\
\hline -T11 \& T12 & \\
\hline
\end{tabular}


Table 2. Radiologic and Clinical Characteristics of Our Treated Patients

\begin{tabular}{|c|c|c|c|}
\hline Index & Preoperative & Immediate Postoperative & Final Follow-Up \\
\hline \multicolumn{4}{|l|}{ Clinical } \\
\hline \multicolumn{4}{|l|}{ ASIA scale } \\
\hline -D, No. (\%) & $5(18.5)$ & $4(14.8)$ & $1(3.7)$ \\
\hline -E, No. (\%) & $22(81.5)$ & $23(85.2)$ & $26(96.3)$ \\
\hline ODI, \%, mean \pm SD (range) & - & - & $15.0 \pm 14.4(0-68)$ \\
\hline VAS, mean $\pm S D$ (range) & - & - & $2.4 \pm 2.5(0-6)$ \\
\hline \multicolumn{4}{|l|}{ Radiological } \\
\hline PTK(Degree) & $+15.7 \pm 3.3($ ranged $;+8$ to +20$)$ & $-8.5 \pm 4.3($ ranged $;-16$ to +1$)$ & $+1 \pm 4.4($ ranged $;-8$ to +10$)$ \\
\hline \multicolumn{4}{|l|}{ Patient satisfaction: } \\
\hline Excellent & - & - & $13(48.1 \%)$ \\
\hline Good & - & - & $11(40.7 \%)$ \\
\hline Fair & - & - & $3(11.1 \%)$ \\
\hline
\end{tabular}

Abbreviations: ODI, oswestry disability index; PTK: post traumatic kyphosis; VAS; visual analogue scale.

diameter or length), and deficient anterior column support (score $>6$ according to McCormack load sharing classification) [23, 24]. Biomechanics' studies have confirmed that screw insertion at the fracture level provide greater construct stability and more resistance to loss of correction $[25,26]$. Eno et al. in a retrospective study reviewed 25 patients with thoracolumbar burst fracture who had been treated with short same segment screw fixation without any supplementary hook [11]. They had two patients (8\%) with revision surgery and reported a LOC of $11.51^{\circ}$ at the 21.6 months follow-up. Mean disability score at the last followup visit was 5.5\% (ranged 0-16\%). They concluded that with short same segment spinal fixation, long-term kyphosis correction was not maintained but this LOC did not correlated with clinical pain and disability. In comparison, we not only involved one more spinal segment in thoracic side of the construct but also used a supplementary infralaminar hook. In our study, no patient required revision surgery and LOC was somewhat lower $\left(9.5 \pm 1.9^{\circ}\right)$ although disability index was higher (15\%). Similar to this study, we also could not find a relationship between LOC and clinical outcome. One of the reasons for the low rate of revision surgery or implant failure in our study is probably due to excluding the patients with more severe injury according to our strict criteria previously mentioned. Tezeren and Kuru in another study on 18 patients with thoracolumbar burst fracture, compare short-segment pedicle fixation with long-segment (two levels above and two levels below) instrumentation [16]. They found that long-segment group had a better outcome at last follow-up visit although there was no difference according to Low Back outcome
Score. Short-segment group had a failure rate of $55 \%$ but shorted operative time and decreased blood loss. Radiologically, long-segment spinal fixation was a more effective treatment, but clinically, surgical outcome was comparable. Similar to our study, these authors could not find an association between clinical outcome of surgery and radiological findings. In order to avoid hard failure complications commonly occur in the patients treated with short segment spinal fixation, Leduc and co-authors tried to use supplementary laminar hooks at both intact upper and lower adjacent vertebrae [27]. In their retrospective study, they have reviewed 25 patients with single level thoracolumbar burst fracture and followed them up for at least one year. Mean LOC at recent follow-up visit was $4^{\circ}$ that was significant for local kyphosis. Similar to our study, instrument failure or pseudoarthrosis did not occur in any patient. Due to short nature of spinal fusion and low rate of surgical complications, the authors recommended this type of surgery for treatment of thoracolumbar burst fractures. Our study had some drawbacks need to be mentioned. First, its retrospective nature inherently carried some faults. Second, we could not compare our results with a long-segment posterior fixation (two levels above and two levels below screw fixation) commonly used by most spinal surgeons. Third, we did not perform computed tomography routinely in our patients to exactly detect any pseudoarthrosis and relied on clinical and radiological evidence. In conclusion, in surgical treatment of thoracolumbar burst fractures, a medium-segment posterior spinal fixation with unilateral same segment pedicle fixation with contralateral infralaminar hook, although 
cannot maintain the radiologic reduction of the fractured vertebrae efficiently, is not only associated with acceptable clinical outcome but also spare one lower lumbar segment and therefore recommended.

\section{Acknowledgments}

The authors wish to thank from deputy of research, Mashhad University of Medical Sciences for financial support through grants no 3054. This paper is based on an orthopedic residency's thesis pertaining to Dr Mahmoud Tavousi.

\section{Footnotes}

Authors' Contributions: The work presented here was carried out in collaboration between all authors: Farzad Omidi Kashani and Ebrahim Ghayem Hasankhani have made substantial contributions to conception and design of the manuscript and have has been involved in drafting the manuscript. Mahmoud Tavousi participated in the sequence alignment. Amir Ashjazadeh has made substantial contributions to acquisition of data from literature. Mohammad Hosein Ebrahimzadeh is the head of research orthopedic center and has made important critical contributions to manuscript revision in terms of its intellectual content.All authors read and approved the final manuscript.

Conflict of Interest: The authors declare that they have no conflict of interests.

Funding/Support: Mashhad University of Medical Sciences, Mashhad, Iran.

\section{References}

1. Wood KB, Li W, Lebl DR, Ploumis A. Management of thoracolumbar spine fractures. Spine J. 2014;14(1):145-64. doi: 10.1016/j.spinee.2012.10.041. [PubMed: 24332321].

2. Ghobrial GM, Jallo J. Thoracolumbar spine trauma: review of the evidence. J Neurosurg Sci. 2013;57(2):115-22. [PubMed: 23676860].

3. Alpantaki K, Bano A, Pasku D, Mavrogenis AF, Papagelopoulos PJ, Sapkas GS, et al. Thoracolumbar burst fractures: a systematic review of management. Orthopedics. 2010;33(6):422-9. doi: 10.3928/0147744720100429-24. [PubMed: 20806752].

4. Wood KB, Buttermann GR, Phukan R, Harrod CC, Mehbod A, Shannon $\mathrm{B}$, et al. Operative compared with nonoperative treatment of a thoracolumbar burst fracture without neurological deficit: a prospective randomized study with follow-up at sixteen to twenty-two years.J Bone Joint Surg Am. 2015;97(1):3-9. doi: 10.2106/JBJS.N.00226. [PubMed: 25568388].

5. Abudou M, Chen X, Kong X, Wu T. Surgical versus non-surgical treatment for thoracolumbar burst fractures without neurological deficit. Cochrane Database Syst Rev. 2013(6):CD005079. doi: 10.1002/14651858.CD005079.pub3. [PubMed: 23740669].
6. Ghobrial GM, Maulucci CM, Maltenfort M, Dalyai RT, Vaccaro AR, Fehlings MG, et al. Operative and nonoperative adverse events in the management of traumatic fractures of the thoracolumbar spine: a systematic review. Neurosurg Focus. 2014;37(1):E8. doi: 10.3171/2014.4.FOCUS1467. [PubMed: 24981907].

7. Thomas KC, Bailey CS, Dvorak MF, Kwon B, Fisher C. Comparison of operative and nonoperative treatment for thoracolumbar burst fractures in patients without neurological deficit: a systematic review. J Neurosurg Spine. 2006;4(5):351-8. doi: 10.3171/spi.2006.4.5.351. [PubMed: 16703901].

8. Lee CS, Chung SS, Shin SK, Park SJ, Lee HI, Kang KC. Differences in postoperative functional disability and patient satisfaction between patients with long (three levels or more) and short (less than three) lumbar fusions. J Bone Joint Surg Br. 2011;93(10):1400-4. doi: 10.1302/0301620X.93B10.27099. [PubMed: 21969442].

9. Kose KC, Inanmaz ME, Isik C, Basar H, Caliskan I, Bal E. Short segment pedicle screw instrumentation with an index level screw and cantilevered hyperlordotic reduction in the treatment of type-A fractures of the thoracolumbar spine. Bone Joint J. 2014;96-B(4):541-7. doi: 10.1302/0301-620X.96B4.33249. [PubMed: 24692625].

10. Wang C, Yang MX, Weng W, Wu AM, Luo P, Chi YL. [Successful short-segment fixation for thoracolumbar burst fractures using CYLpedicle screw]. Zhonghua Wai Ke Za Zhi. 2012;50(1):19-22. [PubMed: 22490284].

11. Eno JJ, Chen JL, Mitsunaga MM. Short same-segment fixation of thoracolumbar burst fractures. Hawaii J Med Public Health. 2012;71(1):19-22. [PubMed: 22413100].

12. Tang J, Liu Y, Hu Y, Cao Z, Lu X, Lin B. Anterior decompression with single segmental spinal interbody fusion for Denis type $B$ thoracolumbar burst fracture: a midterm follow-up study. Int Orthop. 2013;37(11):2205-9. doi: 10.1007/s00264-013-2095-y. [PubMed: 24162182].

13. Schnee CL, Ansell LV. Selection criteria and outcome of operative approaches for thoracolumbar burst fractures with and without neurological deficit. J Neurosurg. 1997;86(1):48-55. doi: 10.3171/jns.1997.86.1.0048. [PubMed: 8988081].

14. Alanay A, Acaroglu E, Yazici M, Aksoy C, Surat A. The effect of transpedicular intracorporeal grafting in the treatment of thoracolumbar burst fractures on canal remodeling. Eur Spine J. 2001;10(6):512-6. [PubMed: 11806392].

15. Farcy JP, Weidenbaum M, Glassman SD. Sagittal index in management of thoracolumbar burst fractures. Spine (Phila Pa 1976). 1990;15(9):958-65. [PubMed: 2259988].

16. Tezeren G, Kuru I. Posterior fixation of thoracolumbar burst fracture: short-segment pedicle fixation versus long-segment instrumentation. J Spinal Disord Tech. 2005;18(6):485-8. [PubMed: 16306834].

17. Denis F. The three column spine and its significance in the classification of acute thoracolumbar spinal injuries. Spine (Phila Pa 1976). 1983;8(8):817-31. [PubMed: 6670016].

18. Marino RJ, Barros T, Biering-Sorensen F, Burns SP, Donovan WH, Graves DE, et al. International standards for neurological classification of spinal cord injury. J Spinal Cord Med. 2003;26 Suppl 1:S50-6. [PubMed: 16296564].

19. Knop C, Oeser M, Bastian L, Lange U, Zdichavsky M, Blauth M. [Development and validation of the Visual Analogue Scale (VAS) Spine Score]. Unfallchirurg. 2001;104(6):488-97. [PubMed: 11460453].

20. Fairbank JC, Pynsent PB. The Oswestry Disability Index. Spine (Phila Pa 1976). 2000;25(22):2940-52. [PubMed: 11074683] discussion 2952.

21. Mousavi SJ, Parnianpour M, Mehdian H, Montazeri A, Mobini B. The Oswestry Disability Index, the Roland-Morris Disability Questionnaire, and the Quebec Back Pain Disability Scale: translation and validation studies of the Iranian versions. Spine (Phila Pa 1976). 2006;31(14):E454-9. doi: 10.1097/01.brs.0000222141.61424.f7. [PubMed: 16778675].

22. Wood EG, Hanley EN. Lumbar disc herniation and open limited discectomy: indications, techniques, and results. Oper Tech Orthop. 1991;1(1):23-8. 
23. Esses SI, Botsford DJ, Wright T, Bednar D, Bailey S. Operative treatment of spinal fractures with the AO internal fixator. Spine (Phila Pa 1976). 1991;16(3 Suppl):S146-50. [PubMed: 2028330].

24. McCormack T, Karaikovic E, Gaines RW. The load sharing classification of spine fractures. Spine (Phila Pa 1976). 1994;19(15):1741-4. [PubMed: 7973969].

25. Mahar A, Kim C, Wedemeyer M, Mitsunaga L, Odell T, Johnson B, et al. Short-segment fixation of lumbar burst fractures using pedicle fixation at the level of the fracture. Spine (Phila Pa 1976). 2007;32(14):1503-
7. doi: 10.1097/BRS.ob013e318067dd24. [PubMed: 17572619].

26. Guven O, Kocaoglu B, Bezer M, Aydin N, Nalbantoglu U. The use of screw at the fracture level in the treatment of thoracolum bar burst fractures. J Spinal Disord Tech. 2009;22(6):417-21. doi: 10.1097/BSD.ob013e3181870385. [PubMed: 19652568].

27. Leduc S, Mac-Thiong JM, Maurais G, Jodoin A. Posterior pedicle screw fixation with supplemental laminar hook fixation for the treatment of thoracolumbar burst fractures. Can J Surg. 2008;51(1):35-40. [PubMed: 18248703]. 